\title{
STATE-DEPENDENT DELAYS ASSOCIATED TO THRESHOLD PHENOMENA IN STRUCTURED POPULATION DYNAMICS
}

\author{
MY LHASSAN HBID \\ S.P.D.S.L. Department of Mathematics, \\ Faculty of Sciences, University Cadi Ayyad, B.P. S15 \\ Marrakesh, Morocco \\ h_hbid@menara.ma \\ EVA SÁNCHEZ \\ Depto. Matemáticas, E.T.S.I. Industriales, \\ c. José Gutiérrez Abascal, 2 \\ 28006 Madrid, Spain \\ evamaria.sanchez@upm.es \\ RAFAEL BRAVO DE LA PARRA \\ Depto. Matemáticas, Universidad de Alcalá, \\ 28871 Alcalá de Henares (Madrid), Spain \\ rafael.bravo@uah.es
}

Received 23 November 2005

Revised 26 September 2006

Communicated by P. Auger

\begin{abstract}
The aim of this paper is to put in evidence the onset of state-dependent delays in threshold models for structured population dynamics. A unified approach to these models is provided, based on solving the corresponding balance law (hyperbolic P.D.E.) along the characteristic lines and showing the common underlying ideas. Size and agestructured models in different fields are presented: insect populations, cell proliferation and epidemics.
\end{abstract}

Keywords: State-dependent delays; thresholds; size and age structured population dynamics.

AMS Subject Classification: 34K60, 35Q80, 37L05

\section{Introduction}

In this work we are interested in the role of state-dependent delayed differential equations in the modeling of structured population dynamics. The aim of this paper is to put in evidence the onset of state-dependent delays in an important class of models: threshold models. These are structured population dynamics models in 
which the transition of individuals between different stages is due to some quantity reaching a prescribed level. An interesting presentation of this kind of equations can be found in Ref. 32 .

Although the appearance of differential equations with state-dependent delays goes back to the 19th century, the study of them has recently been re-activated due to their frequent occurrence in a wide variety of situations. These equations can be found in the literature associated with a wealth of applications in different fields, such as electrodynamics, ${ }^{12}$ economics, ${ }^{6}$ control $^{34}$ and population dynamics. In this context, the state-dependent delay is usually motivated by phenomenological considerations; for example, in Refs. 1 and 4 such a delay is justified as a response of the maturation processes to density-dependence. See also Ref. 5 for a short survey of applications.

Introducing a state-dependent delay in modeling real phenomena results frequently from an attempt to account for the influence of some facts on the behavior of a population. Nevertheless, it is difficult to evaluate the actual impact of a statedependent delay on the response of the model, in particular in what concerns the instability due to oscillations. In fact, these equations pose problems even with regard to the resolution and uniqueness of solutions, as one of the great difficulties in studying them is the impossibility of describing a unified class sufficiently wide to be able to include a large amount of those equations which appear in the models. Some early surveys which deserve mention are Refs. 14 and 23.

When the biology involves thresholds, mathematical difficulties appear in the modeling, which can frequently be solved by placing the problem in the context of functional equations. Our main aim in this paper is to show that some compartmental models can be reduced in a natural way to a particular type of functional equations called threshold type. This situation occurs when the time of residence of the individuals in each compartment is not constant but is fixed by the demand that individuals store a threshold amount of some substance. Usually the rate of accumulation of this substance depends on some state variables, giving rise to statedependent delayed differential equations. This idea has the advantage of permitting the model to be analyzed through the application of a mathematical theory which is currently highly developed. Suitable references are Refs. 13, 19, 31 and 32. The recent work in the theoretical development of state-dependent delayed differential equations is highly developed in Ref. 15 with special emphasis on both the modeling and the emerging theory from the point of view of dynamical systems, as well as an excellent and updated reference list.

From a mathematical point of view, it is well known that structured models of population dynamics which are formulated in terms of hyperbolic partial differential equations can be reduced, integrating along the characteristic lines, to a so-called renewal equation for the function of births. This process, applied to the reduction of a structured model of a population divided into several stages leads frequently to a delayed equation with a time-dependent delay. This variable delay corresponds to the time spent by the individuals of one stage in passing to the next one. If this 
transition depends on the density of the population, then the delay will be statedependent. In many cases, as we will show in the examples below, this condition of transition is formulated like an equation which implicitly defines the delay and which has a meaning in the model: the individuals change their stage after a certain quantity reaches a predetermined threshold value.

In some situations, the magnitude which determines the change of stage depends on the state of a non-structured population $x(t)$, and therefore the threshold condition is formulated as

$$
\int_{t-\tau(t)}^{t} k(x(\sigma)) d \sigma=W^{*}
$$

where $k: \mathbb{R}_{+} \rightarrow \mathbb{R}_{+}$is a known function. There are also other situations where the population is structured by some character in all the stages. In this case, the threshold condition should be expressed in terms of a functional $F$ :

$$
\int_{t-\tau(t)}^{t} F(l(\cdot, \sigma)) d \sigma=W^{*} .
$$

The organization of the paper is as follows: In Sec. 2 we describe the process of appearence of state-dependent delays in size structured models. We illustrate the underlying ideas presenting models for the dynamics of insect populations and cell proliferation. Section 3 is dedicated to age-structured models, describing a process of modeling which can be useful in very general situations. Finally, in Sec. 4 we mention some models in epidemiology, a field which for its importance and development deserves an independent view.

\section{Size Structured Models}

In Ref. 21 the process of modeling the dynamics of populations structured by some physiological variable is described, along with a great amount of examples and applications. With the aim of simplifying, and since it is the context in which the following models are presented, we stick to the one-dimensional case. Let us consider a population in which individuals are determined by a structure variable $x$ (DNA content, maturity, etc.), which we refer to as size and can vary in some interval $\left[x_{b}, x_{d}\right]$. The population is described by a density function $n(x, t)$, such that

$$
\begin{aligned}
\int_{x_{1}}^{x_{2}} n(x, t) d x= & \text { Number of individuals which at instant } t \text { have } \\
& \text { their size } x \text { in the interval }\left[x_{1}, x_{2}\right] .
\end{aligned}
$$

As is fully explained in Ref. 21, the model for the dynamics of this population is obtained through making a balance of mass in $\left[x_{b}, x_{d}\right]$, giving rise to a transport P.D.E.:

$$
\frac{\partial n}{\partial t}(x, t)+\frac{\partial}{\partial x}[g(x, t) n(x, t)]=\text { Sources }- \text { Sinks }
$$


together with a boundary condition which takes into account the entrance of individuals across the boundary $x_{b}$ :

$$
g\left(x_{b}, t\right) n\left(x_{b}, t\right)=\text { Local arrival rate. }
$$

The function $g(x, t)$ is the individual growth rate, so that the individual state is determined by the O.D.E.:

$$
x^{\prime}(t)=g(x, t) ; \quad x(0)=x_{0} .
$$

It is well known that solving (2.1) along the characteristic lines transforms the problem into a renewal equation obtained from (2.2) (see Refs. 36 and 17). This equation involves a variable delay $\tau(x, t)$ which is the time spent by an individual to reach the state $x$ at time $t$ and, therefore, is determined by the equation

$$
x_{b}+\int_{t-\tau(x, t)}^{t} g(x(\sigma), \sigma) d \sigma=x,
$$

where $x(\sigma)$ is the characteristic line through $(x, t)$.

Let us assume that the population is organized in different stages, say juvenile $(\mathrm{J})$ and adult (A) to simplify, and the transition of an individual from (J) to (A) is made when its size reaches a predetermined threshold value $W^{*}>x_{b}$. Then the entrance to the adult stage will depend on $n\left(W^{*}, t\right)$. In turn, calculating this value for $t$ large enough along the corresponding characteristic line, we get an expression in terms of the density $n\left(x_{b}, \tilde{t}\right)$, for some $\tilde{t}<t$. As a consequence, a variable delay $\tau(t)=t-\tilde{t}$ defined by

$$
x_{b}+\int_{t-\tau(t)}^{t} g(x(\sigma), \sigma) d \sigma=W^{*}
$$

will appear in the model. This threshold condition, in those cases where the growth rate $g$ depends on the population, produces in the model a state-dependent delay.

In the models below we illustrate this idea in different situations. We present a classical model by Nisbet and Gurney that develops completely a formalism to deal with insect populations in which the duration of their different instars is variable and is determined by obtaining a predetermined weight. We also applies these ideas to the modeling of cell populations.

\subsection{Insect population dynamics with varying instar duration}

It is an accepted fact that in many insect populations, the passage from one stage to another is not linked to the chronological age, but is produced when the individual reaches a certain weight. Reference 24 is a description of a formalism that allows the modeling of these populations incorporating the variability of the different instars and also providing a mechanism that regulates the growth rate of the adults according to the available food. 
An age and mass dependent population dynamics is described by a density function $\rho(a, m, t)$, so that

$$
\begin{aligned}
\int_{a_{1}}^{a_{2}} \int_{m_{1}}^{m_{2}} \rho(a, m, t) d a d m= & \text { Number of individuals which have ages } a \in\left[a_{1}, a_{2}\right] \\
& \text { and masses } m \in\left[m_{1}, m_{2}\right] \text { at time } t .
\end{aligned}
$$

The dynamics of such a population is described by a balance law:

$$
\frac{\partial \rho}{\partial t}+\frac{\partial \rho}{\partial a}+\frac{\partial}{\partial m}[g \rho]=-\delta \rho,
$$

where $g(a, m, t)$ and $\delta(a, m, t)$ are respectively the rate of growth of an individual and the instantaneous per capita death rate.

The model must be completed with a renewal equation:

$$
\rho(0, m, t)=\int_{0}^{+\infty} \int_{0}^{+\infty} \beta(a, \sigma, m, t) \rho(a, \sigma, t) d a d \sigma
$$

in which $\beta(a, \sigma, m, t)$ is the per capita rate of fecundity.

In the particular situation where $\beta, g$ and $\delta$ are independent of the age, this model can be simplified by integrating over all ages, leading to a model in the new unknown density function

$$
n(m, t):=\int_{0}^{+\infty} \rho(a, m, t) d a .
$$

Assuming that all the individuals have the same mass $m_{b}$ at birth (so that $\rho(0, m, t)=0$ for $\left.m \neq m_{b}\right)$, the balance law is

$$
\frac{\partial n}{\partial t}+\frac{\partial}{\partial m}[g n]=-\delta n, \quad m>m_{b}
$$

The renewal equation (2.3) must be substituted by a boundary condition in $m=m_{b}$. The rate of production of eggs is

$$
E(t)=\int_{m_{b}}^{+\infty} \beta(\sigma, t) n(\sigma, t) d \sigma
$$

and assuming that the survivorship and the duration of the egg stage are constants $P_{E}>0$ and $\tau_{E}>0$ respectively, the rate of recruitment in the first stage of larvae is, for $t>\tau_{E}$ :

$$
R(t)=P_{E} \int_{m_{b}}^{+\infty} \beta\left(\sigma, t-\tau_{E}\right) n\left(\sigma, t-\tau_{E}\right) d \sigma .
$$

This means that $R(t) d t$ is the total recruitment in a time interval $[t, t+d t]$ and that these individuals have their masses in the interval $\left[m_{b}, m_{b}+d m\right]$, with $d m=$ $g\left(m_{b}, t\right) d t$. Since the total number of individuals with masses in $\left[m_{b}, m_{b}+d m\right]$ is $n\left(m_{b}, t\right) d m$, we have

$$
R(t) d t=n\left(m_{b}, t\right) d m=n\left(m_{b}, t\right) g\left(m_{b}, t\right) d t
$$


from which we obtain the boundary condition for the model, for $t>\tau_{E}$ :

$$
g\left(m_{b}, t\right) n\left(m_{b}, t\right)=P_{E} \int_{m_{b}}^{+\infty} \beta\left(\sigma, t-\tau_{E}\right) n\left(\sigma, t-\tau_{E}\right) d \sigma .
$$

Let us consider models for insect populations structured into two stages: larva (L) and adult (A). In what follows we assume that within each stage all individuals have the same growth rate: $g_{L}(t)$ (in $L$ stage) and $g_{A}(t)$ (in $A$ stage), the same per capita death rate: $\delta_{L}(t)$ ( $L$ stage) and $\delta_{A}(t)$ ( $A$ stage) and the same fecundity: $\beta_{L}(t)$ ( $L$ stage) and $\beta_{A}(t)$ ( $A$ stage). We further assume that larvae pass to the adult stage when their weight reaches a threshold value $W^{*}>m_{b}$.

Hence we can assume that stages $(A)$ and $(L)$ can be determined in terms of fixed mass levels and that individuals within each stage can be considered as functionally identical. In consequence we can simplify the model (2.4)-(2.6) deriving a lumped parameter representation (see Ref. 24) for the total populations of larvae and adults, defined by:

$$
L(t):=\int_{m_{b}}^{W^{*}} n(\sigma, t) d \sigma, \quad A(t):=\int_{W^{*}}^{+\infty} n(\sigma, t) d \sigma .
$$

As we will see, this new model allows the description of the population dynamics in terms of delay differential equations which can be better dealt with mathematically.

The dynamics is described by

$$
\left\{\begin{array}{l}
L^{\prime}(t)=R(t)-M_{L}(t)-\delta_{L}(t) L(t) \\
A^{\prime}(t)=\alpha M_{L}(t)-\delta_{A}(t) A(t)
\end{array}\right.
$$

In this system, $R(t)$ is the rate of production of viable eggs at time $t$, which for $t>\tau_{E}$ is defined by $(2.5) ; M_{L}(t)$ is the rate of maturation from $L$ stage and the constant $0 \leq \alpha \leq 1$ is the probability of success of maturation.

First of all, let us observe that $R(t)$ can be expressed in terms of the state variables $L(t), A(t)$ :

$$
R(t)=P_{E} \beta_{L}\left(t-\tau_{E}\right) \int_{m_{b}}^{W^{*}} n\left(\sigma, t-\tau_{E}\right) d \sigma+P_{E} \beta_{A}\left(t-\tau_{E}\right) \int_{W^{*}}^{+\infty} n\left(\sigma, t-\tau_{E}\right) d \sigma
$$

that is

$$
R(t)=P_{E} \beta_{L}\left(t-\tau_{E}\right) L\left(t-\tau_{E}\right)+P_{E} \beta_{A}\left(t-\tau_{E}\right) A\left(t-\tau_{E}\right) .
$$

The main point consists of obtaining the maturation $M_{L}(t)$ in terms of $L(t), A(t)$. Since $M_{L}(t)$ can be viewed as the local arrival rate of Eq. (2.2), we have

$$
M_{L}(t)=g_{L}(t) n\left(W^{*}, t\right)
$$

and then the problem is reduced to calculating $n\left(W^{*}, t\right)$. To this end, we solve the problem (2.4)-(2.6) along the characteristic lines. The characteristic line through $m=m_{b}, t=0$, is

$$
m=m_{b}+\int_{0}^{t} g_{L}(\sigma) d \sigma
$$


Therefore, assuming the existence and uniqueness of $t^{*}>0$, solution to the equation

$$
m_{b}+\int_{0}^{t^{*}} g_{L}(\sigma) d \sigma=W^{*}
$$

straightforward calculations which are not reproduced here lead to the solution, for $t>t^{*}:$

$$
n\left(W^{*}, t\right)=\frac{R(t-\tau(t))}{g_{L}(t-\tau(t))} e^{-\int_{t-\tau(t)}^{t} \delta_{L}(\sigma) d \sigma},
$$

where $\tau(t)$ (which exists and is unique for $t>t^{*}$ ) is the solution to the threshold condition:

$$
m_{b}+\int_{t-\tau(t)}^{t} g_{L}(\sigma) d \sigma=W^{*} .
$$

Notice that the delay $\tau(t)$ is the duration of the larval stage for larvae becoming adults at time $t$.

Summing up, we have obtained the model

$$
\left\{\begin{array}{l}
L^{\prime}(t)=R(t)-R(t-\tau(t)) \frac{g_{L}(t)}{g_{L}(t-\tau(t))} e^{-\int_{t-\tau(t)}^{t} \delta_{L}(\sigma) d \sigma}-\delta_{L}(t) L(t), \\
A^{\prime}(t)=\alpha R(t-\tau(t)) \frac{g_{L}(t)}{g_{L}(t-\tau(t))} e^{-\int_{t-\tau(t)}^{t} \delta_{L}(\sigma) d \sigma}-\delta_{A}(t) A(t)
\end{array}\right.
$$

where $R(t)$ is given by $(2.7)$ and $\tau(t)$ is the solution to (2.8).

In conclusion, the model consists of a system of delayed differential equations with a time-dependent delay defined by the threshold condition (2.8).

The state-dependent delay appears when the growth rate $g_{L}$ depends on the state variable. A simple way can be to introduce a larval competition as

$$
g_{L}(t)=\frac{K_{1}}{K_{2}+L(t)} .
$$

A more complex dependence can be found in Refs. 24, 18 and 3. Let us suppose that larvae live in an environment supplied with food $F(t)$ at a rate $G_{L}(t)$, which is consumed at a rate per larva $A_{L}(t)$, so that the dynamics of the food is described by the O.D.E.:

$$
F^{\prime}(t)=G_{L}(t)-L(t) A_{L}(t)=G_{L}(t)-L(t) \frac{A^{*} F(t)}{K_{F}+F(t)},
$$

where we have accepted a hyperbolic dependence of food consumption rate on food supply (Ref. 24), that is

$$
A_{L}(t)=\frac{A^{*} F(t)}{K_{F}+F(t)} .
$$

Assuming a direct proportionality between larval growth and food assimilitation rates, we have

$$
g_{L}(t)=a A_{L}(t)=\frac{a A^{*} F(t)}{K_{F}+F(t)} .
$$


Since $F(t)$ depends on $L(t), g_{L}$ is density-dependent and therefore $\tau(t)$ is a statedependent delay.

A recent modification of this model focused on the effect of competition between different stages and its effect on population dynamics can be found in Ref. 28. In this model it is assumed that both larvae and adults consume food from the same source $F(t)$. Larvae consume food at a rate $a F(t),(a>0)$ and use that food to increase their weight with a conversion factor $\varepsilon_{g}>0$, such that

$$
g_{L}(t):=\varepsilon_{g} a F(t) .
$$

For their part, adults consume food with a rate $q a F(t),(q>0)$ and use that food for reproduction with a conversion factor $\varepsilon_{b}>0$, such that

$$
\beta_{A}(t):=\varepsilon_{b} q a F(t) .
$$

The mortality of both stages is assumed to be inversely proportional to the ingested food, meaning

$$
\delta_{L}(t):=\frac{\mu}{a F(t)}, \quad \delta_{A}(t):=\frac{\mu}{q a F(t)} .
$$

The total rate of reproduction is

$$
R(t)=\int_{W^{*}}^{+\infty} \beta_{A}(t) n(\sigma, t) d \sigma=\varepsilon_{b} q a F(t) A(t)
$$

and hence the model is modified as follows:

$$
\left\{\begin{aligned}
L^{\prime}(t)= & \varepsilon_{b} q a F(t) A(t)-\frac{F(t)}{F(t-\tau(t))} R(t-\tau(t)) e^{-(\mu / a) \int_{t-\tau(t)}^{t}(d \sigma / F(\sigma))} \\
& -\frac{\mu}{a F(t)} L(t) \\
A^{\prime}(t)= & \alpha \frac{F(t)}{F(t-\tau(t))} R(t-\tau(t)) e^{-(\mu / a) \int_{t-\tau(t)}^{t}(d \sigma / F(\sigma))} \\
& -\frac{\mu}{q a F(t)} A(t)
\end{aligned}\right.
$$

where $R(t)$ is defined by (2.9), together with the O.D.E. which describes the dynamics of the food:

$$
F^{\prime}(t)=D-a F(t) L(t)-q a F(t) L(t)
$$

in which $D>0$ is a constant that represents the rate of replenishment of the resource.

The delay $\tau(t)$ is the solution to the equation

$$
m_{b}+\int_{t-\tau(t)}^{t} \varepsilon_{g} a F(\sigma) d \sigma=W^{*}
$$

and hence it is state-dependent since $F(t)$ depends on both populations $L(t)$ and $A(t)$. 


\subsection{A $G-M$ model in cell kinetics}

Let us consider a population of cells proliferating by division, with the cell cycle divided into two phases: G-phase (resting phase) and M-phase (mitosis). We assume that cells in the G-phase are identical and we denote their number at time $t$ by $P(t)$. The population of cells in the M-phase is structured by the maturity $x(0 \leq x \leq 1)$, $n(x, t)$ being its density so that

$$
\begin{aligned}
\int_{x_{1}}^{x_{2}} n(x, t) d x= & \text { Number of cells in the phase } M \text { with } \\
& \text { maturity } x \in\left[x_{1}, x_{2}\right] \text { at time } t .
\end{aligned}
$$

Maturity is a rather abstract unspecified character associated with a variable $x \in[0,1]$ which marks the progression of an individual cell through the cell cycle (Refs. 9, 22 and 29). A cell entering the M-phase has maturity $x=0$ and all cells divide when they reach maturity $x=1$.

We assume that the maturity of an individual in the M-phase increases deterministically according to the O.D.E:

$$
x^{\prime}(t)=V(P(t))
$$

in which the growth rate $V$ depends on $t$ through $P(t)$. See Ref. 21 for the details.

The model reads as:

$$
\left\{\begin{array}{l}
P^{\prime}(t)=-(\mu+\gamma) P(t)+2 V(P(t)) n(1, t), \\
\frac{\partial n}{\partial t}(x, t)+V(P(t)) \frac{\partial n}{\partial x}(x, t)=-\delta n(x, t),
\end{array}\right.
$$

where $\mu, \gamma, \delta$ are positive constants.

These equations are supplemented by boundary and initial conditions. For individuals in the M-phase we have

$$
V(P(t)) n(0, t)=\gamma P(t), \quad n(x, 0)=n_{0}(x) .
$$

For the G-phase we have $P(0)=P_{0}$.

Solving the equation for $n(x, t)$ along the characteristic lines we obtain for $t$ large enough:

$$
n(x, t)=n(0, t-\tau(x, t)) e^{-\delta \tau(x, t)},
$$

where the delay $\tau(x, t)$ is the solution to the equation

$$
\int_{t-\tau(x, t)}^{t} V(P(\sigma)) d \sigma=x
$$

We will use this expression to calculate $n(1, t)$ which, bearing in mind $(2.10)$, can be expressed in terms of the state variable $P(t)$. Therefore the model is reduced to the following delayed differential equation for $P(t)$ :

$$
P^{\prime}(t)=-(\mu+\gamma) P(t)+2 \gamma \frac{V(P(t)) P(t-\tau(t))}{V(P(t-\tau(t)))} e^{-\delta \tau(t)},
$$


where $\tau(t)$ is defined by the threshold condition

$$
\int_{t-\tau(t)}^{t} V(P(\sigma)) d \sigma=1 .
$$

Differentiating in (2.11) we obtain for the delay an O.D.E. whose coefficients depend on $P(t)$, which makes quite clear that this is a state-dependent delay:

$$
\tau^{\prime}(t)=1-\frac{V(P(t))}{V(P(t-\tau(t)))} .
$$

Notice that $\tau(t)$ is the duration of the M-phase and this duration depends on the total number of cells in G-phase at time $t$.

\subsection{Age-structured models for erythropoiesis}

Biological reasons fully discussed in Refs. 7 and 20 justify the election of an agestructured model for erythropoiesis. In this model two types of cells are considered: precursor $(\mathrm{P})$ and mature $(\mathrm{M})$. The population of precursor cells mature at a variable rate depending on the concentration of Epo. The latter varies according to the oxygen-carrying capacity of the blood. In turn, this depends on (M) cells, which are age-structured.

Let $p(\mu, t)$ be the density of $(\mathrm{P})$ cells which are cells that proliferate and mature after differentiation from the stem cells. The variable $\mu \in\left[0, \mu_{F}\right]$ represents the level of maturity (e.g. content in haemoglobin for the erythrocites), where $\mu_{F}>0$ is the maximum level for a cell reaching maturity. The rate of maturation $V$ of $(\mathrm{P})$ cells depends on the level of hormones $E(t)$, in such a way that high concentrations of Epo accelerate the process. Therefore for the $(\mathrm{P})$ cells, $V$ increases with $E$.

Let $m(a, t)$ be the density of $(\mathrm{M})$ cells, non-proliferating mature cells. The variable $a \in\left[0, a_{F}\right]$ represents the age (with a constant maximum age $a_{F}>0$ ) and it is assumed that the growth rate is a constant $W>0$. The total population of mature cells

$$
M(t):=\int_{0}^{a_{F}} m(a, t) d a
$$

determines the level of the $E$ hormone, in such a way that the concentration of $E$ reduces with high values of $(\mathrm{M})$.

With the aim of writing the balance law for each of the populations, it is assumed that $\beta(\mu, E)$ is the net birth rate for $(\mathrm{P})$, that the rate of mortality of $(\mathrm{P})$ cells is very low so that we do not include such a term in the model, although an incorporation of $(\mathrm{P})$ cells to $(\mathrm{M})$ compartment is produced according to some disappearance rate function $H(\mu)$. We also assume that the death rate of mature cells is $\gamma(a)$, depending only on age. Therefore the model is

$$
\left\{\begin{array}{l}
\frac{\partial p}{\partial t}(\mu, t)+V(E) \frac{\partial p}{\partial \mu}(\mu, t)=\beta(\mu, E) p(\mu, t)-H(\mu) V(E) p(\mu, t), \\
\frac{\partial m}{\partial t}(a, t)+W \frac{\partial m}{\partial a}(a, t)=-\gamma(a) m(a, t)
\end{array}\right.
$$


together with the boundary conditions

$$
V(E) p(0, t)=S_{0}(E), \quad W m(0, t)=\int_{0}^{\mu_{F}} \alpha(\mu) p(\mu, t) d \mu,
$$

where $S_{0}(E)$ is the number of cells recruited into $(\mathrm{P})$ population and $\alpha(\mu)$ reflects the fact that when $(\mathrm{P})$ cells achieve a certain level of maturity, they are released into the bloodstream as (M) cells.

The hormone level $E$ is governed by the O.D.E.:

$$
E^{\prime}(t)=f(M(t))-k E(t)
$$

where $k>0$ is the decay constant for the hormone and $f(M)$ is a monotone decreasing function of $M$.

Solving both P.D.E. along the characteristic lines we obtain (see Ref. 7 for the details):

(a) Let $t_{F}=a_{F} / W$. Then, for $t>t_{F}$, we have:

$$
\begin{aligned}
m(a, t) & =m(0, t-a / W) e^{-\int_{0}^{a} \frac{\gamma(\sigma)}{W} d \sigma} \\
& =\frac{1}{W}\left(\int_{0}^{\mu_{F}} \alpha(\mu) p(\mu, t-a / W) d \mu\right) e^{-\int_{0}^{a} \frac{\gamma(\sigma)}{W} d \sigma .}
\end{aligned}
$$

(b) Let $t_{F}^{*}$ be the solution to the equation $\int_{0}^{t_{F}^{*}} V(E(\sigma)) d \sigma=\mu_{F}$. Then, for $t>t_{F}^{*}$ we have

$$
p(\mu, t)=B(t-\tau(\mu, t)) e^{\int_{t-\tau(\mu, t)}^{t} \mathcal{F}\left(\mu^{*}(\sigma), E(\sigma)\right) d \sigma},
$$

where

- $\tau(\mu, t)$ is the solution to the threshold condition

$$
\int_{t-\tau(\mu, t)}^{t} V(E(\sigma)) d \sigma=\mu
$$

- $\mathcal{F}(\mu, E):=\beta(\mu, E)-V(E) H(\mu)$

- $\mu^{*}(\sigma):=\int_{t-\tau(\mu, t)}^{\sigma} V(E(w)) d w$.

In (a) we have taken into account the second boundary condition of (2.12) and in (b) we have introduced the notation $B(t):=p(0, t)$.

In turn, the first boundary condition of (2.12) transforms (2.15) into

$$
p(\mu, t)=\frac{S_{0}(E(t-\tau(\mu, t)))}{V(E(t-\tau(\mu, t)))} e^{\int_{t-\tau(\mu, t)}^{t} \mathcal{F}\left(\mu^{*}(\sigma), E(\sigma)\right) d \sigma} .
$$

Finally, for $t$ large enough $\left(t>\max \left(t_{F}, t_{F}^{*}\right)\right)$, let us consider the O.D.E. (2.13) for the dynamics of $E(t)$. Bearing in mind (2.14) we obtain

$$
M(t)=\frac{1}{W} \iint_{\left[0, a_{F}\right] \times\left[0, \mu_{F}\right]} \alpha(\mu) p(\mu, t-a / W) e^{-\int_{0}^{a} \frac{\gamma(\sigma)}{W} d \sigma} d a d \mu .
$$

Substituting into this expression the corresponding formula for $p(\mu, t-a / W)$ obtained from (2.15) and then substituting $M(t)$ in Eq. (2.13), we have reduced 
the problem to a delayed differential equation for $E(t)$, in which the delay $\tau(\mu, t)$ is defined by the threshold condition (2.16) which involves $E(t)$, so that it is a state-dependent delay.

The underlying ideas in this model are similar to the ones before, but show a greater complexity because the threshold value $\mu$ is variable. In this model the transition of $(\mathrm{P})$ cells to $(\mathrm{M})$ does not happen when the $(\mathrm{P})$ cells reach a predetermined level of maturity, but when they reach a certain variable level $\mu$ according to a given distribution $\alpha(\mu)$.

An extension of this model to include the active destruction of the oldest mature cells and possible control by apoptosis is developed in Ref. 20. This results in a moving boundary condition in which $a_{F}(t)$ varies with $t$ :

$$
\left(W-a_{F}^{\prime}(t)\right) m\left(a_{F}(t), t\right)=Q,
$$

where $Q>0$ is a fixed removal rate.

The complexity of the formulations obtained in Refs. 7 and 20 make simplifications necessary to cover their mathematical analysis. Following the point of view accepted in Ref. 20, we assume that $V(E)=W=1$, which implies that $\tau(\mu, t)=\mu$. Assume that $(\mathrm{P})$ cells grow exponentially in accordance with

$$
\beta(\mu, E):= \begin{cases}\beta & \text { if } 0<\mu<\tilde{\mu}, \\ 0 & \text { if } \mu \geq \tilde{\mu},\end{cases}
$$

where $\beta>0, \tilde{\mu}>0$ are given constants. Moreover, we will assume that the passage of $(\mathrm{P})$ cells to $(\mathrm{M})$ cells occurs only on the boundary, which means that we change the second condition of (2.12) for the condition

$$
m(0, t)=p(\tilde{\mu}, t) .
$$

Resulting in:

$$
\left\{\begin{aligned}
m(a, t) & =p(\tilde{\mu}, t-a) e^{-\int_{0}^{a} \gamma(\sigma) d \sigma}=S_{0}(E(t-a-\tilde{\mu})) e^{-\int_{0}^{a} \gamma(\sigma) d \sigma}, \\
p(\mu, t) & =S_{0}(E(t-\tilde{\mu})) e^{\beta \tilde{\mu}} .
\end{aligned}\right.
$$

In Ref. 20, the total population of mature cells is given by

$$
M(t)=\int_{0}^{a_{F}(t)} m(a, t) d a,
$$

where $a_{F}(t)$ is defined through the moving boundary condition (2.17). Some simple calculations (see Ref. 20) allow the model to be reduced to the following system of delayed differential equations for the variables $M(t), E(t), a_{F}(t)$, in which a constant delay and a state-dependent delay appear:

$$
\left\{\begin{array}{l}
E^{\prime}(t)=f(M(t))-k E(t), \\
M^{\prime}(t)=-\gamma M(t)+e^{\beta \tilde{\mu}} S_{0}(E(t-\tilde{\mu}))-Q, \\
a_{F}^{\prime}(t)=1-\frac{Q e^{-\beta \tilde{\mu}} e^{\gamma a_{F}(t)}}{S_{0}\left(E\left(t-\tilde{\mu}-a_{F}(t)\right)\right)} .
\end{array}\right.
$$

More recent references for hematopoiesis models can be found in Refs. 10, 11 and 8 . 


\section{Age-Structured Models with Moving Boundary Condition}

As we have seen in the previous section, the delay is the time employed by each individual to reach the threshold value of a certain variable, which means in agestructured models that the age of passing from one stage to the next is timedependent. Therefore, unlike what occurs in size-structured models in which the exit to the following stage is produced for a constant value of the structure variable and the variability is due to the growth rate, in age-dependent models the time employed for each individual to reach the threshold value is the age of exit, which gives place to a moving boundary. The situation is similar to what described in Ref. 20.

To illustrate these ideas, let us consider a population divided into two stages, the juveniles and the adults, each one being structured by the age in the stage. Denote by $l(a, t)$ (resp. $L(a, t))$ the density of juveniles (resp. adults) of age $a$ at time $t$. We have the following equations:

$$
\left\{\begin{array}{c}
\frac{\partial l}{\partial t}(a, t)+\frac{\partial l}{\partial a}(a, t)=-\mu_{l}(a) l(a, t), \\
\frac{\partial L}{\partial t}(a, t)+\frac{\partial L}{\partial a}(a, t)=-\mu_{L}(a) L(a, t),
\end{array}\right.
$$

where $\mu_{l}(a)$ (resp. $\mu_{L}(a)$ ) is the age-dependent mortality rate of juveniles (resp. adults). We point out the fact that the variable $a$ represents the age of juveniles in the function $l(a, t)$ while it is the age of the adults in the function $L(a, t)$.

The above two equations have to be supplemented by boundary conditions and initial values. For the juvenile we have

$$
l(0, t)=B(t), \quad l(a, 0)=0,
$$

where $B(t)$ represents the time density of newborns and the initial condition expresses the fact that no juvenile is supposed to exist at the time $t=0$ (or, no one is able to survive beyond a certain period of time).

The moving boundary is caused by the expression of $B(t)$ in each model. Let us begin with a simple example (see Ref. 3 ).

We define $J(t)$ to be the biomass of juveniles at time $t$, i.e.

$$
J(t):=\int_{0}^{a_{M}} l(a, t) d a
$$

$a_{M}\left(0<a_{M}<+\infty\right)$ being the maximum age of juveniles (i.e. individuals which have not reached the threshold value past time $a_{M}$, will die or never reach the next stage) and let us assume that

$$
B(t)=k J(t), \quad k>0 .
$$

The passage from the juvenile to the adult stage is described in terms of the weight function of the juvenile, namely we assume that

$$
\frac{\partial w}{\partial a}(a, t)+\frac{\partial w}{\partial t}(a, t)=\frac{K}{J(t)+C},
$$


where $w(a, t)$ is the quantity of food eaten until time $t$ by an individual entering the juvenile stage $a$ time units ago. $K>0$ is the quantity of food entering the species habitat per unit of volume and per unit of time, which for simplicity is considered to be a constant. The constant $C>0$ represents the food (converted into a number of individuals) taken per unit of volume by consumers other than juvenile stage. Limitation of food due to density is modeled in the simplest possible way, assuming that the quantity of food available is shared in equal parts by all the individuals occupying the same space at time $t$.

Assuming that $w(0, t)=w(a, 0)=0$ (which corresponds to the absence of juveniles at time $t=0)$, we arrive at

$$
w(a, t)=\int_{t-a}^{t} \frac{K}{J(\sigma)+C} d \sigma .
$$

We now introduce the threshold condition: juveniles turn adult when the food index reaches a prescribed value $W^{*}>0$, that is at each time $t, a^{*}(t)$ is the unique solution to the equation

$$
\int_{t-a^{*}(t)}^{t} \frac{K}{J(\sigma)+C} d \sigma=W^{*}
$$

and so

$$
J(t)=\int_{0}^{a^{*}(t)} l(a, t) d a .
$$

Solving the equation in $l$ from system (3.1) along the characteristic lines starting from $a=0$, we obtain

$$
l(a, t)=B(t-a) e^{-\int_{0}^{a} \mu_{l}(s) d s}=k J(t-a) e^{-\int_{0}^{a} \mu_{l}(s) d s}
$$

which, bearing in mind (3.3) and accepting for simplicity that $\mu_{l}=0$, provides the following equation for $J(t)$ :

$$
J(t)=k \int_{t-a^{*}(t)}^{t} J(\sigma) d \sigma .
$$

Differentiating in (3.2) and (3.4) we reduce the problem to the following system of state-delayed differential equations

$$
\left\{\begin{aligned}
J^{\prime}(t) & =k\left[J(t)-J\left(t-a^{*}(t)\right) \frac{J\left(t-a^{*}(t)\right)+C}{J(t)+C}\right], \\
\left(a^{*}\right)^{\prime}(t) & =\frac{J\left(t-a^{*}(t)\right)-J(t)}{J(t)+C} .
\end{aligned}\right.
$$

Let us describe a more general situation. For the juvenile we consider the renewal equation

$$
l(0, t)=B(t)=\int_{0}^{+\infty} \beta(a) L(a, t) d a,
$$

where $\beta(a)$ is the age-dependent rate of fecundity for the adults. 
For the adult we have

$$
L(a, 0)=L_{0}(a)
$$

and we now introduce a threshold condition: the juveniles turn adult when some variable reaches a prescribed value. Therefore, we can express the fact that $L(0, t)$ depends on $l\left(a^{*}(t), t\right)$, where $a^{*}(t)$ is defined by a threshold condition:

$$
\int_{t-a^{*}(t)}^{t} F(l(\cdot, \sigma)) d \sigma=W^{*},
$$

where $W^{*}>0$ is a prescribed constant. That is, we accept that the evolution of juveniles to the threshold is described by a continuous and positive functional

$$
F: L^{1}\left(\left[0, a_{M}\right]\right) \longrightarrow \mathbb{R} .
$$

Regarding the existence of $a^{*}(t)$, let us observe that for each $t>0$ fixed, the function

$$
g:[0, t] \longrightarrow \mathbb{R}, \quad g(a):=\int_{t-a}^{t} F(l(\cdot, \sigma)) d \sigma
$$

is continuous, strictly increasing, with $g(0)=0$. Therefore, for each $t>0$ fixed, there exists a unique solution $a^{*}(t)$ to (3.5) if and only if $g(t)>W^{*}$. We look for a solution such that $0<a^{*}(t) \leq a_{M}$, hence a necessary and sufficient condition is

$$
\int_{0}^{\min \left(t, a_{M}\right)} F(l(\cdot, \sigma)) d \sigma>W^{*} .
$$

In particular, let us assume that there exists a constant $F^{*}>0$ such that

$$
\forall \varphi \in L^{1}\left(\left[0, a_{M}\right]\right), \quad \varphi \geq 0, \quad 0 \leq F(\varphi) \leq F^{*} .
$$

Then, a necessary condition for the existence of solution to (3.6) is, for $t \geq a_{M}$ :

$$
F^{*} a_{M} \leq W^{*} .
$$

Solving Eq. (3.1) along the characteristic lines, we obtain

$$
l(a, t)= \begin{cases}l(0, t-a) e^{-\int_{0}^{a} \mu_{l}(u) d u} & \text { if } t>a \\ 0 & \text { if } t<a\end{cases}
$$

with

$$
\begin{gathered}
l(0, t-a)=\int_{0}^{+\infty} \beta(\sigma) L(\sigma, t-a) d \sigma, \\
L(a, t)= \begin{cases}L(0, t-a) e^{-\int_{0}^{a} \mu_{L}(u) d u} & \text { if } t>a, \\
L_{0}(a-t) e^{-\int_{a-t}^{a} \mu_{L}(u) d u} & \text { if } t<a .\end{cases}
\end{gathered}
$$

Our aim is to show that the above problem can be transformed into a statedependent delay functional equation for $B(t)$, in which the state-dependence is due to the threshold condition (3.5). 
We have

$$
\begin{aligned}
B(t) & =\int_{0}^{+\infty} \beta(\sigma) L(\sigma, t) d \sigma \\
& =\int_{0}^{t} \beta(\sigma) L(\sigma, t) d \sigma+\int_{t}^{+\infty} \beta(\sigma) L(\sigma, t) d \sigma
\end{aligned}
$$

and, bearing in mind (3.7), we can write

$$
B(t)=\int_{0}^{t} \Pi(\sigma) L(0, t-\sigma) d \sigma+G\left(L_{0}, t\right)
$$

where we have introduced the notations:

$$
\begin{aligned}
\Pi(\sigma) & :=\beta(\sigma) e^{-\int_{0}^{\sigma} \mu_{L}(u) d u} d \sigma \\
G\left(L_{0}, t\right) & :=\int_{t}^{+\infty} \beta(\sigma) L_{0}(\sigma-t) e^{-\int_{\sigma-t}^{\sigma} \mu_{L}(u) d u} d \sigma .
\end{aligned}
$$

Let us observe that $G\left(L_{0}, t\right)$ is a known function, depending on the initial age distribution for the adults.

Proposition 3.1. For the cohorts entering the adult stage we have

$$
L(0, t)=\left[1-\left(a^{*}\right)^{\prime}(t)\right] l\left(a^{*}(t), t\right) .
$$

Proof. The formula is obtained by applying a book-keeping principle to those juveniles which are susceptible to enter the adult stage. At time $t$ their number is given by

$$
N(t)=\int_{0}^{a^{*}(t)} l(\sigma, t) d \sigma .
$$

By differentiating this formula we obtain

$$
\begin{aligned}
N^{\prime}(t) & =l\left(a^{*}(t), t\right)\left(a^{*}\right)^{\prime}(t)+\int_{0}^{a^{*}(t)} \frac{\partial l}{\partial t}(\sigma, t) d \sigma \\
& =l\left(a^{*}(t), t\right)\left(a^{*}\right)^{\prime}(t)-\int_{0}^{a^{*}(t)}\left[\frac{\partial l}{\partial \sigma}(\sigma, t)+\mu_{l}(\sigma) l(\sigma, t)\right] d \sigma \\
& =l(0, t)-l\left(a^{*}(t), t\right)\left[1-\left(a^{*}\right)^{\prime}(t)\right]-\int_{0}^{a^{*}(t)} \mu_{l}(\sigma) l(\sigma, t) d \sigma .
\end{aligned}
$$

The quantity $l(0, t)$ is the flow in, and $l\left(a^{*}(t), t\right)\left[1-\left(a^{*}\right)^{\prime}(t)\right]$ is the flow out, yielding formula (3.9).

Substituting (3.9) into (3.8), we have:

$$
B(t)=\int_{0}^{t} \Pi(\sigma)\left[1-\left(a^{*}\right)^{\prime}(t-\sigma)\right] l\left(a^{*}(t-\sigma), t-\sigma\right) d \sigma+G\left(L_{0}, t\right) .
$$


The solution to (3.5), if it exists, satisfies $a^{*}(t)<t$ and then $\forall \sigma \in[0, t]$ we have $a^{*}(t-\sigma)<t-\sigma$, which yields to

$$
\begin{aligned}
l\left(a^{*}(t-\sigma), t-\sigma\right) & =l\left(0, t-\sigma-a^{*}(t-\sigma)\right) e^{-\int_{0}^{a^{*}(t-\sigma)} \mu_{l}(u) d u} \\
& =B\left(t-\sigma-a^{*}(t-\sigma)\right) e^{-\int_{0}^{a^{*}(t-\sigma)} \mu_{l}(u) d u} .
\end{aligned}
$$

Summing up, we have obtained for $B(t)$ the following equation

$$
\begin{aligned}
B(t)= & \int_{0}^{t} \prod(\sigma)\left[1-\left(a^{*}\right)^{\prime}(t-\sigma)\right] B\left(t-\sigma-a^{*}(t-\sigma)\right) e^{-\int_{0}^{a^{*}(t-\sigma)} \mu_{l}(u) d u} d \sigma \\
& +G\left(L_{0}, t\right) .
\end{aligned}
$$

Furthermore, we deduce from

$$
l(a, t)=H(t-a) B(t-a) e^{-\int_{0}^{a} \mu_{l}(u) d u},
$$

where $H(\cdot)$ is the Heaviside function (i.e. $H(u)=1$ if $u>0, H(u)=0$ if $u<0$ ), that $a^{*}(t)$ can be obtained as the solution to the following equation, depending on $B(t)$ :

$$
\int_{t-a^{*}(t)}^{t} F\left(B(\sigma-\cdot) e^{-\int_{0}^{\cdot} \mu_{l}(u) d u} H(\sigma-\cdot)\right) d \sigma=W^{*} .
$$

We have reached our purpose: $B(t)$ is the solution to (3.10) which is a functional delayed equation with the delay $a^{*}(t)$ being a solution to Eq. (3.11), which depends on $B(t)$. In other words, we have obtained a state-dependent delay functional equation for $B(t)$, starting from a model without delay: obviously the delay arises from the threshold condition. An example of this type of models can be found in Ref. 2.

In our previous situation the functional $F$ is:

$$
F(l(\cdot, \sigma)):=\frac{K}{J(\sigma)+C}=\frac{K}{\int_{0}^{a_{M}} l(a, \sigma) d a+C},
$$

that is:

$$
\text { for all } \varphi \in L^{1}\left(\left[0, a_{M}\right]\right), \quad F(\varphi):=\frac{K}{\int_{0}^{a_{M}} \varphi(a) d a+C} \text {. }
$$

Remark. In some models the passage from one stage to the next is determined by a threshold condition which yields to a delayed equation for $B(t)$ with a timedependent delay, but not a state-dependent delay. For example (see Refs. 26 and 27), in some fish population models the life cycle is divided into three phases: pelagic (larvae), juvenile and adult. The juvenile phase starts when the larvae reach a critical (threshold) size. It is accepted in some cases that the growth in size of larvae is determined by the temperature, so that

$$
w(a, t)=\int_{t-a}^{t} f(T(\sigma)) d \sigma,
$$

where $T(t)$ is the temperature at time $t$ and $f$ is a positive smooth function. 
In this case, the delay $a^{*}(t)$ is the solution to the threshold condition

$$
w(a, t)=W^{*}
$$

and therefore it is not a state-dependent delay.

\section{Threshold State-Dependent Delays in Epidemiology}

State-dependent delay functional differential equations arise in a natural way in epidemiology, when the passage of susceptible individuals to the infected compartment is due to the accumulation of a certain amount of infection. Let us present a typical example of such situation in the following model, whose details can be found in Ref. 30. See also Refs. 15, 16 and 35 for related models.

Let us consider an infectious disease propagating in an isolated population which is divided into four disjoint groups:

- $S:=$ Susceptible individuals which have not been exposed to the infection,

- $E:=$ Individuals who have been exposed to the disease but are not yet infective,

- $I:=$ Infectives,

- $R:=$ Individuals who have recovered and are temporarily immune.

Schematically, the progression of an individual in the process can be represented in the following diagram:

$$
S \rightarrow E \rightarrow I \rightarrow R \rightarrow S .
$$

Let us denote by $S(t), E(t), I(t), R(t)$ the respective total populations of the four groups, at time $t$. The infection is assumed to spread according to the following rules:

(i) The rate of exposure by infectives at time $t$ is given by $r(t) S(t) I(t)$ where the contact rate $r(t)$ is a given positive function.

(ii) An individual who is first exposed at time $\tau(t)$ becomes infective at time $t$ if

$$
\int_{\tau(t)}^{t}\left[\rho_{1}(s)+\rho_{2}(s) I(s)\right] d s=W^{*},
$$

where $\rho_{1}(t), \rho_{2}(t)$ are two given positive functions and $W^{*}>0$ is a predetermined constant.

(iii) An individual which is infected at time $t$ recovers at time $t+\sigma$, where $\sigma>0$ is a given constant.

(iv) An individual, upon recovering, is immune for a time interval of length $w \geq 0$, after which he becomes susceptible again.

(v) The population size remains to be a constant $N>0$, i.e.

$$
S(t)+E(t)+I(t)+R(t)=N .
$$

Condition (ii) is the threshold condition of the present model. It means that the individual can control minor exposure to the infection, i.e. there is a level of 
tolerance in such a way that the immune system can fight exposure below that level. When the exposure is high, the individual is infected. The amount of infection received depends on the duration of the exposure and on the amount of infection surrounding the individual, which in turn is assumed to be proportional to the number of infected individuals in the population (term $\rho_{2}(s) I(s)$ ). The term $\rho_{1}(s)$ is the exposure accumulation rate that does not depend on the number of infectives (e.g. constant entrance of virus from the exterior).

Let us assume that the initial populations of susceptibles $S_{0}$ and infectives $I_{0}$ are given, such that $S_{0}+I_{0}=N$.

In order to build the model we also know the path history of infectives $I_{0}(t)$, $t \in[-\sigma, 0]$, which we will assume is a continuous nondecreasing function such that $I_{0}(0)=I_{0}$. According to the previous rules it is possible to extend this function to $\mathbb{R}_{+}$so that it describes the number of these individuals initially infected that stay in class $I$ for $t \geq 0$ :

$$
I_{0}(t):=I_{0}-I_{0}(t-\sigma), t \in[0, \sigma] ; \quad I_{0}(t)=0, t>\sigma .
$$

A necessary condition for the infection to spread is that some initially susceptible individuals in the population become infected before time $\sigma$, which forces us to assume the existence of a time $t_{0} \in(0, \sigma)$ such that

$$
\int_{0}^{t_{0}}\left[\rho_{1}(s)+\rho_{2}(s) I_{0}(s)\right] d s=W^{*} .
$$

From the previous assumptions the following system of integral equations for the spreading of the disease is obtained:

$$
\left\{\begin{array}{l}
S(t)=I_{1}(t)+S_{0}-\int_{\tau(t-\sigma-w)}^{t} r(s) I(s) S(s) d s, \\
I(t)=I_{0}(t)+\int_{\tau(t-\sigma)}^{\tau(t)} r(s) I(s) S(s) d s, \\
E(t)=\int_{\tau(t)}^{t} r(s) I(s) S(s) d s, \\
R(t)=I_{2}(t)+\int_{\tau(t-\sigma-w)}^{\tau(t-\sigma)} r(s) I(s) S(s) d s,
\end{array}\right.
$$

completed with the threshold condition (4.1).

The functions

$$
\begin{aligned}
& I_{1}(t)= \begin{cases}0 & \text { if } t \leq w \\
I_{0}-I_{0}(t-w) & \text { if } t \geq w\end{cases} \\
& I_{2}(t)= \begin{cases}I_{0}-I_{0}(t) & \text { if } t \leq w \\
I_{0}(t-w)-I_{0}(t) & \text { if } t \geq w\end{cases}
\end{aligned}
$$

represent those individuals initially infected who have passed to the classes $S$ and $R$ respectively. 
Notice that the first two equations of the above system together with the condition (4.1) can be viewed as a state-dependent delayed differential system for $S(t), I(t)$.

\subsection{Infectious diseases in orchards controlled by a "Roguing and Replanting" strategy}

Some structured models in epidemiology fit in our study and can be reduced to a state-dependent delayed differential equation. In this section we present a model for an infectious disease in orchards, controlled by a so-called "Roguing and Replanting" strategy. This model was first proposed and numerically analyzed in Ref. 33 and mathematically developed in Ref. 25. Since it is an age-structured model, it gives place to a moving boundary condition.

To study the dynamics of an epidemic in an orchard, let us assume that infections are localized in leaves or branches called sites. A tree is composed of sites and will be classified according to the number of infected sites on it. The number of infections on a tree increases as a function of the force of infection, due to all the trees being in the orchard. A rogued tree is replaced with an uninfected one, and the criterion for roguing is a fixed threshold number for the infections.

Denote by $L(a, t)$ the number of infected sites on trees of age $a$ at time $t$, by $R(a, t)$ the number of post-infections sites on trees of age $a$ at time $t$ and by $M(a, t)$ the density of trees of age $a$ at time $t$, so that $\int_{a_{1}}^{a_{2}} M(a, t) d a$ is the total number of trees with ages $a \in\left[a_{1}, a_{2}\right]$.

The model reads for $t>0, a>0$, as:

$$
\left\{\begin{aligned}
\frac{\partial L}{\partial t}(a, t)+\frac{\partial L}{\partial a}(a, t) & =\beta I(t)[\alpha-L(a, t)] \\
\frac{\partial R}{\partial t}(a, t)+\frac{\partial R}{\partial a}(a, t) & =\mu[L(a, t)-R(a, t)], \\
\frac{\partial M}{\partial t}(a, t)+\frac{\partial M}{\partial a}(a, t) & =-w M(a, t)
\end{aligned}\right.
$$

where $\alpha, \beta, \mu, w$ are positive constants and $I(t)$ is the total number of infections in the orchard at time $t$, so that

$$
I(t)=\int_{0}^{a_{\max }(t)}[L(a, t)-R(a, t)] M(a, t) d a
$$

in which $a_{\max }(t)$ is the maximum age in the orchard, defined by a threshold infection number $L_{\max }>0$ that satisfies

$$
L\left(a_{\max }(t), t\right)=L_{\max } .
$$

Since a rogued tree is immediately replaced by an uninfected one, we assume that the total number of trees in the orchard is a constant $N>0$ and therefore

$$
\int_{0}^{a_{\max }(t)} M(a, t) d a=N .
$$


The model is completed with boundary and initial conditions respectively:

$$
L(0, t)=R(0, t)=0
$$

and

$$
L(a, 0)=L_{0}(a), \quad R(a, 0)=R_{0}(a), \quad M(a, 0)=M_{0}(a) .
$$

First of all, let us solve Eq. (4.2) along the characteristic lines, taking into account the boundary conditions (4.6). Standard but somewhat lengthy calculations which are not reproduced here lead to:

$$
\begin{aligned}
& L(a, t)= \begin{cases}L_{0}(a-t) e^{-\beta \int_{0}^{t} I(\sigma) d \sigma}+\alpha\left[1-e^{-\beta \int_{0}^{t} I(\sigma) d \sigma}\right] & \text { if } a>t, \\
\alpha\left[1-e^{-\beta \int_{t-a}^{t} I(\sigma) d \sigma}\right] & \text { if } a<t,\end{cases} \\
& R(a, t)= \begin{cases}e^{-\mu t}\left[R_{0}(a-t)+\mu L_{0}(a-t) \int_{0}^{t} e^{\mu \sigma-\beta \int_{0}^{\sigma} I(s) d s} d \sigma\right. \\
\left.+\mu \alpha \int_{0}^{t} e^{\mu \sigma}\left(1-e^{-\beta \int_{0}^{\sigma} I(s) d s}\right) d \sigma\right] & \text { if } a>t, \\
\mu \alpha e^{-\mu a} \int_{0}^{a} e^{\mu \sigma}\left(1-e^{-\beta \int_{t-a}^{t-a+\sigma} I(s) d s}\right) d \sigma & \text { if } a<t,\end{cases} \\
& M(a, t)= \begin{cases}M_{0}(a-t) e^{-w t} & \text { if } a>t, \\
M(0, t-a) e^{-w a} & \text { if } a<t .\end{cases}
\end{aligned}
$$

The case $a_{\max }(t)>t$ says that the maximum age in the orchard at time $t$ is the age of a cohort existing before the initial time $t=0$ that is, the roguing process has not reached all initial trees yet. In what follows we will assume that there exists $t^{*}>0$ such that $a_{\max }\left(t^{*}\right)=t^{*}$ and $\forall t \geq t^{*}, a_{\max }(t) \leq t$. Therefore, we assume that the roguing process will reach all the initial cohorts, which is the case when the maximum age of the orchard cannot exceed a finite value $a_{\max }^{*}>0$ (see Ref. 25 for details). In this case, for $t \geq t^{*}$ conditions (4.5) and (4.3) allow us to reduce the original model to the following system of delayed equations in the unknown functions $I(t), B(t):=M(0, t)$ :

$$
\left\{\begin{array}{l}
\int_{t-a_{\max }(t)}^{t} B(\sigma) e^{w \sigma} d \sigma=N e^{w t} \\
I(t) e^{w t}=\int_{t-a_{\max }(t)}^{t} \mathcal{F}(t, u, I) B(u) e^{w u} d u
\end{array}\right.
$$

where we have used the notation

$$
\mathcal{F}(t, u, I):=-\alpha e^{-\beta \int_{u}^{t} I(\sigma) d \sigma}+\alpha e^{-\mu(t-u)}\left[1+\mu \int_{0}^{t-u} e^{\mu \sigma} e^{-\beta \int_{u}^{u+\sigma} I(s) d s} d \sigma\right] .
$$

In turn, the delay $a_{\max }(t)$ satisfies the threshold condition (4.4), i.e.

$$
\alpha\left[1-e^{-\beta \int_{t-a_{\max }(t)}^{t} I(\sigma) d \sigma}\right]=L_{\max }
$$

and therefore, it is dependent on the state variable $I(t)$ through the threshold condition

$$
\int_{t-a_{\max }(t)}^{t} I(\sigma) d \sigma=I^{*}, \quad I^{*}:=-\frac{1}{\beta} \log \left(1-\frac{L_{\max }}{\alpha}\right)
$$

as we have wanted to prove. 


\section{Conclusion}

In this paper we present a unified approach for size and age-structured population dynamics whose individuals are classified in different stages and the passage from one stage to the next is due to some variable reaching a predetermined value. In these models a balance law formulated as a hyperbolic P.D.E. allows us to reduce the study to the different cohorts, creating a variable delay which is the time spent for individuals in each stage.

As we have put in evidence in Sec. 2, making some simplifying assumptions, sizestructured models can be reduced to compartmental models that consider the population divided into various stages corresponding to size values at different intervals. The passage of individuals from one stage to the following occurs when the structure variable size reaches a threshold value. Therefore, the permanence in each stage is variable and is determined by the growth law which is specified in the model. This means that the variable delay in the formulation is determined by a threshold condition (e.g. (2.11)) in which the growth rate appears. This delay is state-dependent when the growth rate depends on the state variables of the model. Our goal in this section has been to make clear that, though biological processes are very different in each of the examples presented, nevertheless the underlying ideas in the reduction of models initially written in terms of PDEs to systems of functional delayed differential equations, are common. It can be checked in the references given, that this reduction permits a mathematical analysis that provides quite enough information about their behavior: possible oscillations, stationary solutions, etc.

The situation described for age-structured models is slightly different, the main body of this work is in Sec. 3. A process of reduction to delayed differential equations is described, valid for a fairly general situation of an age-structured population. The variability in these models is due to the exit age of each compartment being defined by a threshold condition (3.5) so that the passage to the following compartment occurs through a moving boundary condition (3.9). A next step in our work consists of a detailed mathematical study of the state-dependent delayed model for a fish population constructed in Ref. 2, which will be published elsewhere.

\section{Note Added}

This paper is in loving memory of our friend Ovide Arino, whose suggestions about the subject are the origin of this work.

\section{Acknowledgments}

E.S. and R.B.P. were supported by Ministerio de Educación y Ciencia (Spain), proyecto MTM2005-00423 and FEDER.

\section{References}

1. W. G. Aiello, H. I. Freedman and J. Wu, Analysis of a model representing stagestructured population growth with state-dependent time delay, SIAM J. Appl. Math. 52 (1992) 855-869. 
2. O. Arino, M. L. Hbid and R. Bravo de la Parra, A mathematical model of growth of population of fish in the larval stage: Density-dependence effects, Math. Biosci. 150 (1998) 1-20.

3. O. Arino and E. Sánchez, Delays induced in population dynamics, in Mathematical Modeling of Population Dynamics, Banach Center Publications, Vol. 63 (Polish Acad. Sci., 2004), pp. 9-46.

4. O. Arino, E. Sánchez and A. Fathallah, State-dependent delay differential equations in population dynamics: Modeling and analysis, Field Inst. Commun. 29 (2001) 19-36.

5. J. Bélair, Population models with state-dependent delays, in Mathematical Population Dynamics, Lecture Notes in Pure and Applied Mathematics, Vol. 131, eds. O. Arino, D. Axelrod and M. Kimmel (Marcel-Dekker, 1991), pp. 165-176.

6. J. Bélair and M. C. Mackey, Consumer memory and price fluctuations on commodity markets: an integrodifferential model, J. Dyn. Diff. Eqns. 1 (1989) 299-325.

7. J. Bélair, M. C. Mackey and J. M. Mahaffy, Age-structured and two-delay models for erythropoiesis, Math. Biosci. 128 (1995) 317-346.

8. J. Bélair and J. M. Mahaffy, Variable maturation velocity and parameter sensitivity in a model of hematopoiesis, IMA J. Math. Appl. Med. Biol. 18 (2001) 193-211.

9. N. Bellomo, A. Bellouquid and M. Delitala, Mathematical topics on the modeling complex multicellular systems and tumour immune cells competition, Math. Mod. Meth. Appl. Sci. 14 (2004) 1683-1733.

10. C. Colijn and M. C. Mackey, A mathematical model of hematopoiesis I: Periodic chronic myelogenous leukemia, J. Theor. Biol. 237 (2005) 117-132.

11. C. Colijn and M. C. Mackey, A mathematical model of hematopoiesis II: Cyclical neutropenia, J. Theor. Biol. 237 (2005) 133-146.

12. R. D. Driver, A "backwards" two-body problem of classical relativistic electrodynamics, Phys. Rev. 178 (1969) 2051-2057.

13. J. A. Gatica and P. Waltman, Existence and uniqueness of solutions of a functional differential equation modeling thresholds, Nonlinear Anal. Th. Meth. Appl. 8 (1984) $1215-1222$.

14. H. Halanay and J. A. Yorke, Some new results and problems in the theory of differential-delay equations, SIAM Rev. 13 (1971) 55-80.

15. F. Hartung, T. Krisztin, H.-O. Walther and J. Wu, Functional differential equations with state-dependent delays: Theory and applications, in Handbook of Differential Equations: Ordinary Differential Equations, Vol. 3, eds. A. Canada, P. Drabek and A. Fonda (North-Holland, 2006).

16. F. Hoppenstead and P. Waltman, A problem in the theory of epidemics, I, II, Math. Biosci. 9 (1971) 71-91; 12 (1970) 133-145.

17. M. Iannelli, Mathematical Theory of Age-Structured Population Dynamics, Appl. Math. Monographs, Vol. 7 (Giardini Editori e Stampatori in Pisa, 1994).

18. A. E. Jones, R. M. Nisbet, W. S. C. Gurney and S. P. Blythe, Period to delay ratio near stability boundaries for systems with delayed feedback, J. Math. Anal. Appl. 135 (1988) 354-368.

19. Y. Kuang and H. L. Smith, Slowly oscillating periodic solutions of autonomous statedependent delay equations, Nonlinear Anal. Th. Meth. Appl. 19 (1992) 885-872.

20. J. M. Mahaffy, J. Bélair and M. C. Mackey, Hematopoietic model with moving boundary condition and state dependent delay, J. Theor. Biol. 190 (1998) 135-146.

21. J. A. J. Metz and O. Diekmann, The Dynamics of Physiologically Structured Populations, Lecture Notes in Biomath. Vol. 68 (Springer-Verlag, 1986).

22. S. Mischler, B. Perthame and L. Ryzhik, Stability in a nonlinear population maturation model, Math. Mod. Meth. Appl. Sci. 12 (2002) 1751-1772. 
23. A. D. Myshkis, On certain problems in the theory of differential equations with deviating arguments, Usp. Mat. Nauk 32 (1977) 173-202 [English translation, Russ. Math. Surv. 32 (1977) 181-213].

24. R. M. Nisbet and W. S. C. Gurney, The systematic formulation of population models for insects with dynamically varying instar duration, Theor. Popul. Biol. 23 (1983) $114-135$.

25. A. Ramzi, Well-posedness of a model of population dynamics of infectious diseases with "Roguing and Replanting" as strategy of control, J. Biol. Sys. 5 (1997) 403-431.

26. A. Ramzi, O. Arino, C. Koutsikopoulos, A. Boussoir and P. Lazure, Modeling and numerical simulations of the sole (Solea Solea (L.)) of the Bay of Biscay. Part 1: Modeling, Oceanol. Acta 24 (2001) 101-112.

27. A. Ramzi, O. Arino, C. Koutsikopoulos, A. Boussoir and P. Lazure, Modeling and numerical simulations of the sole (Solea Solea (L.)) of the Bay of Biscay. Part 2: Numerical simulations, Oceanol. Acta 24 (2001) 113-124.

28. A. M. de Roos and L. Persson, Competition in size-structured populations: Mechanisms inducing cohort formation and population cycles, Theor. Pop. Biol. 63 (2003) $1-16$.

29. S. I. Rubinow, A maturity-time representation for cell populations, Biophys. J. 8 (1968) 1055-1070.

30. H. Smith, Hopf bifurcation in a system of functional equations modeling the spread of an infectious disease, SIAM J. Appl. Math. 43 (1983) 370-385.

31. H. Smith, Reduction of structured population models to threshold-type delay equations and functional differential equations: A case study, Math. Biosci. 113 (1993) 1-23.

32. H. L. Smith and Y. Kuang, Periodic solutions of differential delay equations with threshold-type delays, Contemp. Math. 129 (1992) 153-176.

33. F. Van den Bosch and A. M. de Roos, The dynamics of infectious deseases in orchards with "Roguing and Replanting" as control strategy, Technical Note (1994).

34. H. O. Walther, Stable periodic motion of a system with state-dependent delay, Diff. Integral Eqns. 15 (2002) 923-944.

35. P. Waltman, Deterministic Threshold Models in the Theory of Epidemics, Lecture Notes in Biomath, Vol. 1 (Springer-Verlag, 1974).

36. G. F. Webb, Theory of Nonlinear Age-Dependent Population Dynamics, Pure and Appl. Math., Vol. 89 (Marcel-Dekker, 1985). 